\title{
Study on Cost Evaluation of DC Smart Houses
}

\author{
Kentaro Shimomachia,,, Yuji Mishima ${ }^{\text {a }}$

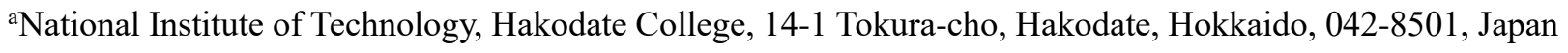 \\ *Corresponding Author: shimomachi@hakoadte-ct.ac.jp
}

\begin{abstract}
Smart house, which is installed on-site distributed generators like photovoltaics and batteries and manages their own energy usage by using information technology, is studied and developed all over the world. Most of facilities in a smart house are operated in DC systems; however, conventional supply systems are AC. In addition, electric vehicles which also need DC power have been increasing in residential side. Therefore, a DC power supply system for smart houses can be reduce the number of power converters, conversion losses and electric charge. Initial and running costs of DC-based smart houses and AC-based one are calculated in several conditions in this paper. In conclusion, DC-based smart houses require lower costs than AC-based one regardless of $\mathrm{AC} / \mathrm{DC}$ ratio in load and uses of $\mathrm{EV}$.
\end{abstract}

Keywords: smart house, DC power supply, cost reduction.

\section{Introduction}

In recent years, studies which aim to disseminate renewable energy (RE) like photovoltaics (PVs) and wind energy conversion systems are increasing. Most of outputs of generation with RE fluctuates due to effect of weather, time and etc.. An installation of energy storage systems (ESSs) like a battery is one of method to control RE outputs $^{(1)}$. By expanding this method, smart grid (SG) which can correct and process information from facilities, namely RE outputs data, ESS data and load data is increasing $^{(2)(3)}$. Moreover, the idea of smart house (SH) which apply this idea to not only a power system but also individual house is also increasing. For example, a home energy management system (HMES) was proposed in (4), and it can enhance energy efficiency in SH.

On the other hand, conventional power system supply AC power to demands. Therefore, PVs and ESSs are needed power converters to interconnect power system and them because they output DC power. On the demand side, many electric appliances consume DC power. Thus, outputs from DC source must go through DC/AC converters and $\mathrm{AC} / \mathrm{DC}$ converters even if demands need DC power. This fact shows that it is uneconomical to directly install DC source for DC loads. In (5), reductions of the number of converters and conversion losses by installing DC power supply system at a data center which has larger amount of DC loads than residences. Moreover, high efficiency energy supply method in a $\mathrm{SH}$ which has only DC loads is proposed in (6). In a case of that mixed AC and DC loads remain in $\mathrm{SH}$, it was shown that ratio of $\mathrm{DC}$ loads can determine cost reduction in (7). In addition, electric vehicles (EVs) which also use DC power are increasing in residential side. Therefore, DC power supply in SH will be required to achieve high efficiency energy usage in the future. In (7), two types of plural SHs connection are connected; SHs connected to conventional AC girds independently and small DC network containing SHs connected to grids are evaluated in economical point of view. PVs and ESSs are considered in (7); however, EVs are not considered. Therefore, economic evaluation of power supply forms in SHs which include EV operation patterns are proposed in this paper.

\section{Outline of SH Group}

\subsection{Structure of SH group}

In this paper, it is assumed that five SHs make a group and they are connected to same feeder line as shown in Fig. 1 and 2. A group that every $\mathrm{SH}$ is connected to AC line independently called ACSH group, and small DC network containing SHs is called DCSH group in this paper. Every SH has AC load, DC load, a PV, a EV and a battery in both cases. Although electricity from each SH can flow into utility grid in principles, reverse power flow from $\mathrm{SH}$ is inhibited to avoid the situation that SHs earn electricity 


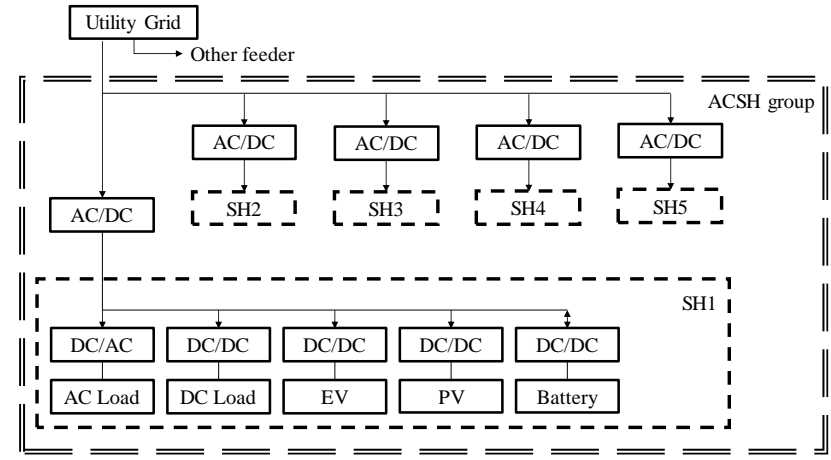

Fig. 1. Structure of ACSH group.

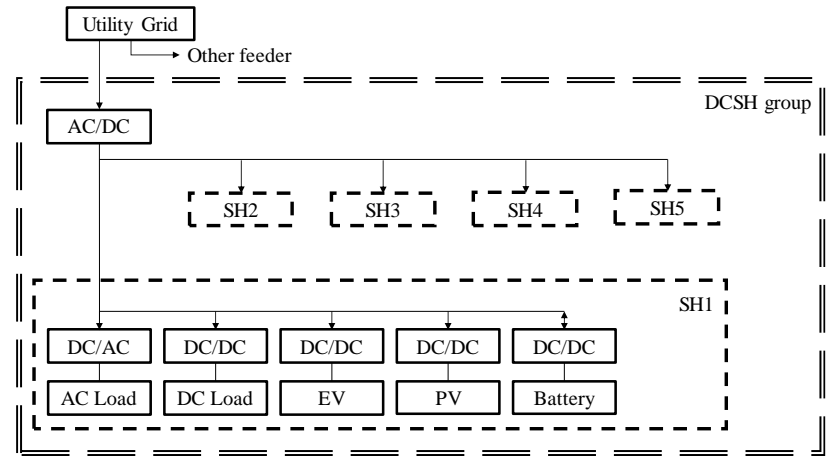

Fig. 2. Structure of DCSH groun.

benefit by exploiting TOU costs. Capacity of PVs and batteries are determined by solve optimization problems shown in next section.

\subsection{Problem Formulation}

In this paper, capacity of PVs, batteries and interfacing converter are optimized to minimize total cost including initial costs and running costs in SH groups. Therefore, the objective function in this problem can be written as follows:

$$
\min f=I C_{P V}+I C_{\text {batt }}+I C_{\text {int }}+R C
$$

where each IC indicates initial cost of each facilities [JPY], namely, PVs, batteries and interfacing converters. $R C$ is running cost of SH groups during a day [JPY]. Particularly, each cost can be written as follows:

$$
\begin{gathered}
I C_{P V}=\sum_{n=1}^{5} C_{P V} \cdot \operatorname{Cap} p_{P V}^{n} \\
I C_{\text {batt }}=\sum_{n=1}^{5} C_{\text {batt }} \cdot \operatorname{Cap}_{\text {batt }}^{n} \\
I C_{\text {conv }}=\sum_{n=1}^{5} C_{\text {conv }} \cdot \operatorname{Cap_{\text {conv}}^{n}} \\
R C=\sum_{n=1}^{5} \sum_{t=1}^{24} \operatorname{TOU}(t) \cdot P_{\text {grid }}^{n}(t) \cdot \Delta T
\end{gathered}
$$

where each $C$ is unit initial cost $[\mathrm{JPY} / \mathrm{kW}$ ], each Cap is capacity of the facility in $n$th SH [kW]. In case of ACSH group, $I C_{\text {conv }}$ must be written in a summation form as (4) because each SH has own interfacing converter. TOU $(t)$ is electricity price at time $t[\mathrm{JPY} / \mathrm{kWh}], P_{\text {grid }}^{n}(t)$ is purchasing power of $n$th SH from utility grid (in case of ACSH group) $[\mathrm{kW}], P_{\text {grid }}(t)$ is purchasing power of DCSH group [kW], $\Delta T$ is duration in numerical simulation [h]. In this paper, a day is partitioned into 24 durations and $\Delta T$ must be $1.0[\mathrm{~h}]$. In case of DCSH group, a interfacing converter is integrated. Thus, $I C_{\text {conv }}$ and $R C$ can be written as (4)' and (5)' respectively.

$$
\begin{gathered}
I C_{\text {conv }}=C_{\text {conv }} \cdot \operatorname{Cap}_{\text {conv }} \\
R C=\sum_{t=1}^{24} T O U(t) \cdot P_{g r i d}(t) \cdot \Delta T
\end{gathered}
$$

Constraints to solve the problems will be written as follows.

(a) Power balance constraint

Summation of power flow supply from the grid and/or the facilities must equal to load, which may contain charging power of EV and battery. This constraint must be satisfied in any durations and any SHs.

$$
\begin{aligned}
& P_{\text {grid }}^{n}(t)+P_{P V}^{n}(t)+P_{b a t t_{D}}^{n}(t) \\
& \quad=P_{A C L}^{n}(t)+P_{D C L}^{n}(t)+P_{E V_{C}}^{n}(t)+P_{\text {batt }_{C}}^{n}(t)
\end{aligned}
$$

Here, $P^{n}(t)$ is power flow of each facility [kW], suffix batt $_{C}$ and batt $_{D}$ indicate charging and discharging respectively. This constraint can be rewritten in case of DCSH group because reverse power flow from one SH to another SH is not inhibited. Thus, the constraint is applied to the network under the interfacing converter. This means power flow from each SH can be collected as follows.

$$
\begin{aligned}
& P_{\text {grid }}(t)+\sum_{n=1}^{5} P_{P V}^{n}(t)+P_{\text {batt }_{D}}^{n}(t) \\
& =\sum_{n=1}^{5} P_{A C L}^{n}(t)+P_{D C L}^{n}(t)+P_{E V_{C}}^{n}(t)+P_{b a t t_{C}}^{n}(t)
\end{aligned}
$$

(b) Capacity constraint

Power flow of each facilities cannot exceed their own $\mathrm{kW}$ capacity limitation.

$$
\begin{aligned}
& P_{x x}(t)<P_{x x}^{\max } \\
& P_{x x}(t)>P_{x x}^{\min }
\end{aligned}
$$

Here, suffix $x x$ identify the facility, $\min$ and $\max$ indicate downer limitation and upper limitation.

(c) Battery charging and discharging constraint

In general, a battery cannot charge and discharge simultaneously. To avoid such situation in simulations, 
binary (0-1) variables which indicate state of battery are introduced. Thus capacity constraints of a battery should be rewritten as follows.

$$
\begin{aligned}
& P_{\text {batt }}^{\min } \leq P_{\text {batt }_{C}}^{n}(t) \leq P_{\text {batt }}^{\max } \cdot\left\{1-u_{\text {batt }}^{n}(t)\right\} \\
& P_{\text {batt }}^{\min } \leq P_{\text {batt }}^{n}(t) \leq P_{\text {batt }}^{\max } \cdot u_{\text {batt }}^{n}(t)
\end{aligned}
$$

If $u_{\text {batt }}^{n}(t)=0$, battery can charge and cannot discharge and vice versa.

(d) Constraint concerned state of charge (SOC)

SOC of battery depends on its previous state. The unit is $\mathrm{kWh}$.

$$
\begin{aligned}
& \operatorname{SOC}_{\text {batt }}^{n}(t+1) \\
& \quad=\operatorname{SOC}_{\text {batt }}^{n}(t)+\left\{P_{\text {batt }_{C}}^{n}(t)-P_{\text {batt }}^{n}(t)\right\} \cdot \Delta T
\end{aligned}
$$

On the other hand, EVs have also their own battery. Thus, SOC of EV can be written as follows, similarly.

$$
\begin{aligned}
& \operatorname{SOC}_{E V}^{n}(t+1) \\
& =S_{O C} C_{E V}^{n}(t)+\left\{P_{E V_{C}}^{n}(t)-P_{E V_{D}}^{n}(t)\right\} \cdot \Delta T
\end{aligned}
$$

However, it is assumed that EVs cannot discharge their SOC for energy supply. Thus, $P_{E V_{D}}(t)$ is just used for their drive in this paper. Both SOC are limited between downer and upper limit at any times.

$$
\begin{aligned}
& S O C_{\text {batt }}^{n}(t)<S O C_{\text {batt }}^{n \max } \\
& S O C_{\text {batt }}^{n}(t)>S O C_{\text {batt }}^{n \text { min }} \\
& S O C_{E V}^{n}(t)<S O C_{E V}^{n \max } \\
& S O C_{E V}^{n}(t)>S O C_{E V}^{n} \min
\end{aligned}
$$

In general, the upper limit of SOC depends on their $\mathrm{kW}$ capacity (2.2 times in this paper)

(e) $\mathrm{CO}_{2}$ emission reduction constraint

One of effects of $\mathrm{SH}$ introduction is $\mathrm{CO}_{2}$ emission reduction. The government of Japan sets $\mathrm{CO}_{2}$ emission reduction goal: $26 \%$ referenced on 2013 . Thus, SHs should contribute for reduction, not only for their own aim. Here, it is assumed that $\mathrm{SHs}$ should achieve $\mathrm{CO}_{2}$ emission reduction as follows.

$$
\frac{\mathrm{CO}_{2}^{\text {base }}-\mathrm{CO}_{2}}{\mathrm{CO}_{2}^{\text {base }}} \geq 0.26
$$

Here, $\mathrm{CO}_{2}^{\text {base }}$ is referenced value $[\mathrm{kg}]$ and $\mathrm{CO}_{2}$ is calculated $\mathrm{CO}_{2}$ emission from $\mathrm{SHs}[\mathrm{kg}]$. Equation (18) is for ACSH group and (18)' for DCSH group.

$$
\begin{gathered}
\mathrm{CO}_{2}=\sum_{n=1}^{5} \sum_{t=1}^{24} C O_{2}^{\text {unit }}(t) \cdot P_{\text {grid }}^{n}(t) \\
\mathrm{CO}_{2}=\sum_{t=1}^{24} C O_{2}^{\text {unit }}(t) \cdot P_{\text {grid }}(t)
\end{gathered}
$$

Finally, decision variables in this problem are as follows; $\operatorname{Cap}_{P V}^{n}, \operatorname{Cap}_{\text {batt }}^{n}$, either $\operatorname{Cap}_{\text {conv }}^{n}$ or $\operatorname{Cap}_{\text {conv }}$, either $P_{\text {grid }}^{n}(t)$ or $P_{\text {grid }}(t), P_{\text {batt }_{C}}^{n}(t), P_{\text {batt }_{D}}^{n}(t)$, and $u_{\text {batt }}^{n}(t)$. This problem is $0-1$ linear problem because all decision variables but $u_{b a t t}^{n}(t)$ are real number, and all constraints are linear.

\section{Simulations}

To evaluate ACSH group and DCSH group, a day of summer are employed for numerical simulation. In this paper, two case are simulated. Firstly, ratio of DC load are changed from $0 \%$ to $100 \%$. This simulation does not contain EV. Then, another case which considered various operation patterns of EV for fixed DC load ratio are simulated. The given data in both simulations are shown in Table.1. These are same value in (7). Demand data and PV output unit data are shown in Fig.3.

\subsection{Effects of Ratio of DC}

Total costs of ACSH and DCSH group are shown in Fig. 4. Ratio of DC load is horizontal axis. In every ratio, DCSH group is lower than ACSH. Especially, in case of $40 \%$, a gap of cost become largest and its value is 4913 [JPY]. Installed capacities in case of $40 \%$ are shown in Table 2. Outputs of each facilities are shown in Fig. 5 and 6. Change of SOC for each SH group is shown in Fig.7. First, the capacity of battery for DCSH is smaller than ACSH one. Second, SOC of battery reached upper limitation during daytime. PV outputs are larger than demand in each case due to $\mathrm{CO}_{2}$ emission reduction constraints. Thus, surplus power must be absorbed by battery or share to another $\mathrm{SH}$. However, ACSH group cannot select to share power, battery must be large.

\subsection{Effects of EV operation patterns}

This section describes about effects of EV operation patterns to simulation results. In all simulation in this section, DC load ratio is fixed to $40 \%$. Three EV operation patterns, use to go to office/school, to go to shopping and to go to a trip are assumed in this paper ${ }^{(9)}$. All patterns are shown in Table 3. In case of A, an EV will leave $\mathrm{SH}$ at 8:00 and will return at 19:00. It runs $40 \mathrm{~km}$ during the trip. In case of B, an EV will leave SH at 13:00 and will return at 17:00. It runs $15 \mathrm{~km}$ during the trip. In case of C, an EV will leave $\mathrm{SH}$ at 14:00 and will return at 22:00. It runs 160 $\mathrm{km}$ during the trip. After the trip, EV will charge to recover SOC.

Total costs in each case are shown in Fig. 8. The costs for all patters but case 1 increased because the EVs charged after the trip. It pushed up electric demand and running cost 
Table 1. Given data in a simulation.

\begin{tabular}{|c|c|}
\hline Character & Value \\
\hline$C_{P V}$ & $31.9[\mathrm{JPY} / \mathrm{kW}]$ \\
\hline$C_{\text {batt }}$ & $51.0[\mathrm{JPY} / \mathrm{kW}]$ \\
\hline$C_{\text {conv }}$ & $13.7[[\mathrm{JPY} / \mathrm{kW}]$ \\
\hline \multirow{2}{*}{$T O U(t)$} & $28.08[\mathrm{JPY} / \mathrm{kWh}](7: 00-23: 00)$ \\
\cline { 2 - 2 } & $14.13[\mathrm{JPY} / \mathrm{kWh}]($ Other $)$ \\
\hline \multirow{2}{*}{$C O_{2}^{\text {unit }}(t)$} & $0.690[\mathrm{~kg} / \mathrm{kWh}](6: 00-18: 00)$ \\
\cline { 2 - 2 } & $0.469[\mathrm{~kg} / \mathrm{kWh}]($ Other $)$ \\
\hline$P_{E V}^{\min }$ & $0.0[\mathrm{~kW}]$ \\
\hline$P_{E V}^{\max }$ & $3.0[\mathrm{~kW}]$ \\
\hline$S O C_{E V}^{\min }$ & $0[\mathrm{kWh}]$ \\
\hline$S O C_{E V}^{\max }$ & $12.8[\mathrm{kWh}]$ \\
\hline & \\
\hline
\end{tabular}

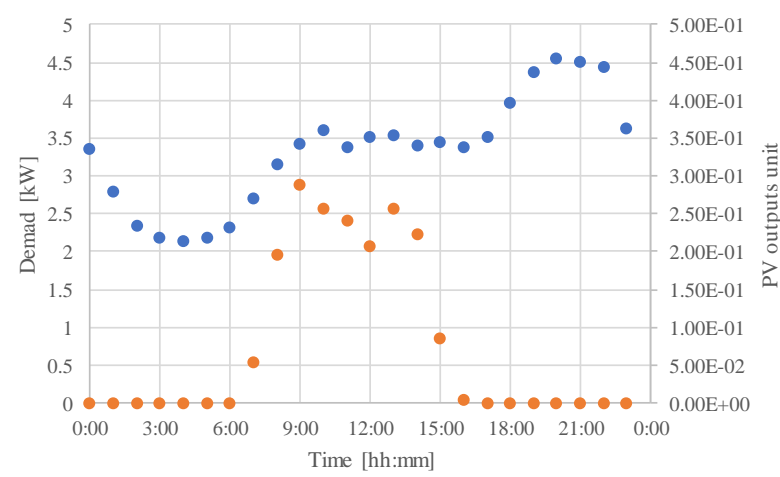

Fig. 3. Demand an PV data.

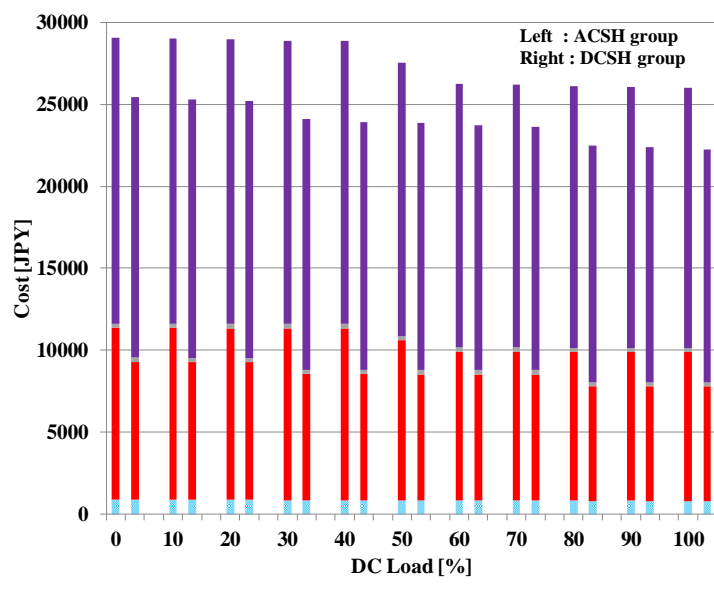

$=$ PV $=$ Battery $=$ Converter $=\mathbf{R C}$

Fig. 4. Total cost comparison between ACSG and DCSH

Table 2. Capacity of facilities.

\begin{tabular}{|c|c|c|}
\hline & ACSH & DCSH \\
\hline PV & $30.0[\mathrm{~kW}]$ & $30.0[\mathrm{~kW}]$ \\
\hline Battery & $45.0[\mathrm{~kW}]$ & $33.0[\mathrm{~kW}]$ \\
\hline Converter & $18.92[\mathrm{~kW}]$ & $18.92[\mathrm{~kW}]$ \\
\hline
\end{tabular}

of SH. However, in every case, the costs of DCSH group

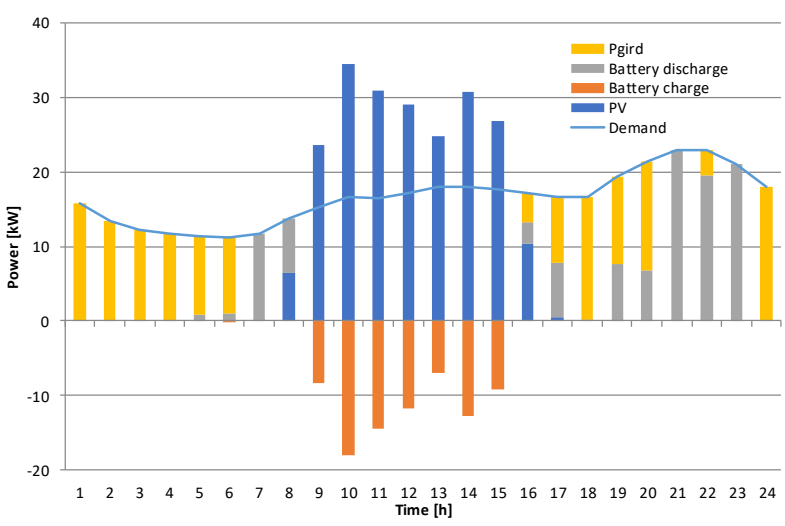

Fig. 5. Operation of ACSH group

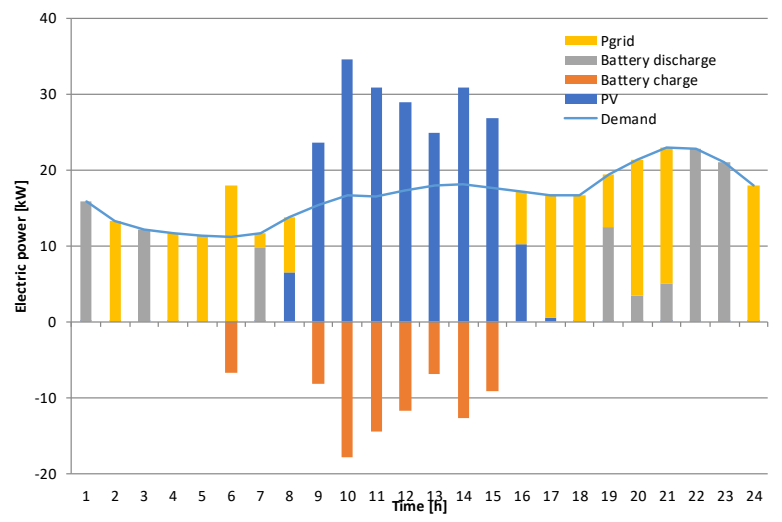

Fig. 6. Operation of DCSH group

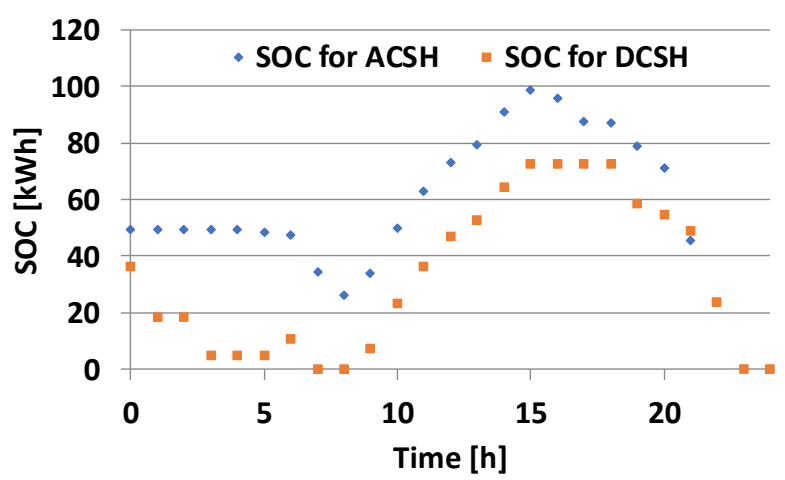

Fig. 7. Change of SOC

are lower than ACSH. Conclusion, DCSH can reduce total costs in every case assumed in this paper.

\section{Conclusions}

Economic evaluation of power supply forms in SHs which include $\mathrm{EV}$ operation patterns are proposed in this paper. Firstly, effects of DC load ratio were compared between two types of SH group. DCSH group which can share surplus power among SHs can realize lower costs than ACSH group. Second, effects of plural EV operation patterns were evaluated. DCSH can reduce total costs in 
Table 3. EV operation patterns.

\begin{tabular}{|c|c|c|c|c|c|c|}
\hline Pattern & Category & \multicolumn{5}{|c|}{ Purpose } \\
\hline 1 & No EV & - & - & - & - & - \\
\hline 2 & All for A & A & A & A & A & A \\
\hline 3 & All for B & B & B & B & B & B \\
\hline 4 & All for C & $\mathrm{C}$ & $\mathrm{C}$ & $\mathrm{C}$ & C & $\mathrm{C}$ \\
\hline 5 & Varying & $\mathrm{C}$ & - & - & - & - \\
\hline 6 & & $\mathrm{C}$ & $\mathrm{C}$ & - & - & - \\
\hline 7 & & $\mathrm{C}$ & $\mathrm{C}$ & C & - & - \\
\hline 8 & & $\mathrm{C}$ & $\mathrm{C}$ & $\mathrm{C}$ & C & - \\
\hline 9 & Town & A & A & A & B & B \\
\hline 10 & City & A & A & B & B & $\mathrm{C}$ \\
\hline 11 & Metropolis & A & A & B & B & - \\
\hline
\end{tabular}

every pattern assumed in this paper.

Furthermore, larger SH group will be considered, and network construction costs will be calculated.

\section{Acknowledgment}

A part of this work is performed by Kazuki Narita who has graduated Advanced Course at National Institute of Technology, Hakodate College.

\section{References}

(1) Sonal Gaurav, Chirag Birla, Aman Lamba, S. Umashankar and Swaminathan Ganesan : "Energy Management of PV - Battery Based Microgrid System”, Procedia Technology, Vol. 21, pp. 103-111, 2015

(2) Yeliz Yoldaş, Ahmet Önen, S. M. Muyeen, Athanasios V. Vasilakos and İrfan Alana : "Enhancing smart grid with microgrids: Challenges and opportunities", Renewable and Sustainable Energy Reviews, Vol. 72, pp. 205-214, 2017

(3) Ikuo Kurihara : "Power System Technology to Realize Smart Grid”, IEEJ Transactions on Power and Energy, Vol. 133, No. 4, pp.298-301, 2013 (in Japanese)

(4) Mohammad Shakeri, Mohsen Shayestegan, S. M. Salim Reza, Iskandar Yahya, Badariah Bais, Md Akhtaruzzaman, Kamaruzzaman Sopiand and Nowshad Amin : "Implementation of a novel home energy management system (HEMS) architecture with solar photovoltaic system as supplementary source", Renewable Energy, Vol. 125, pp. 108-120, 2018

(5) Keiichi Hirose : "Trends of DC Power Technologies

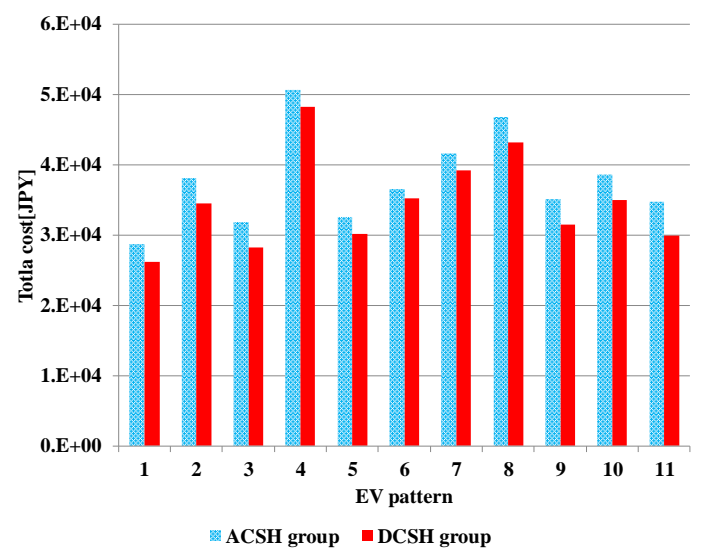

Fig. 8. Total costs comparison with EV patterns.

and their Applications", IEEJ Transactions on Power and Energy, Vol. 131, No. 4, pp.358-361, 2011 (in Japanese)

(6) Kenichi Tanaka, Akihiro Yoza, Kazuki Ogimi, Atsushi Yona, Tomonobu Senjyu, Toshihisa Funabashi and Chul-Hwan Kim : "Optimal operation of DC smart house system by controllable loads based on smartgrid topology", Renewable Energy, Vol. 39, pp. 132-139, 2012

(7) Kentaro Shimomachi, Ryoichi Hara and Hiroyuki Kita : "Comparison between DC and AC Microgrid Systems Considering Ratio of DC Load”, IEEE PES Asia-Pacific Power and Energy Engineering Conference 2015 (IEEE PES APPEEC 2015) , PES-APPEEC 062, 2015 